\title{
Anisotropic nanocomposite hydrogels with enhanced actuating performance through aligned polymer networks
}

\author{
Ping Tang ${ }^{1 \dagger},{\text { Hao } \text { Yan }^{2,4 \dagger} \text {, Lie Chen }}^{2,5}$, Qingshan $\mathrm{Wu}^{2}$, Tianyi Zhao ${ }^{2}$, Shuhong $\mathrm{Li}^{1^{*}}$, Hainan Gao ${ }^{1}$ and \\ Mingjie $\mathrm{Liu}^{2,3 *}$
}

\begin{abstract}
Anisotropic composite hydrogels have wide applications in the fields of materials for actuators and sensors. Herein, we report an anisotropic composite hydrogel prepared by a mechanical-strain-induced method. Polymer networks including poly $(N$-isopropylacrylamide) (PNIPAM) and sodium alginate (SA), as well as carbon nanotubes (CNTs) are found to align simultaneously by stretching, and then fixed by physical crosslinking through non-covalent bonds. Composite hydrogels with doubly aligned polymer networks showed anisotropic optical and mechanical properties. The actuation performance of the anisotropic composite hydrogels as compared with the isotropic ones was found to be enhanced, which showed the capability of lifting 100 times its weight with $20 \%$ contraction strain. Besides, a bilayer hydrogel was designed to bend with a maximum of $390^{\circ}$ to mimic the tendril behavior of plants.
\end{abstract}

Keywords: anisotropic, PNIPAM-based hydrogels, interpenetrating polymer network, actuation

\section{INTRODUCTION}

Hydrogels with ordered anisotropic structures [1,2], similar to biological tissues [3-6], have promising applications in the fields of actuators and sensors [7-9]. To build the anisotropic structure in hydrogels, self-assembly through intermolecular interactions was used [10], and external field effects, such as electrical field [11], magnetic field [12-17], mechanical-strain-induced (stretch) [18-
28], shear-force-induced $[29,30]$ and directional freezing [31], have been employed. Currently, hydrogels prepared by the above mentioned methods achieve anisotropic properties [7-9], such as anisotropic optical properties [14,22,32], mechanical performances [18], actuating abilities [14,33] and electrical conductivity [11].

Poly ( $N$-isopropylacrylamide) (PNIPAM) is the most typical stimuli-responsive polymer and can be extensively used as a building block of hydrogels. PNIPAM could provide the thermal-actuated functionality, as it exhibits a reversible volume phase transition at a lower critical solution temperature $\left(\mathrm{LCST} \sim 32^{\circ} \mathrm{C}\right.$ ) [34-38]. As for the PNIPAM-based hydrogel actuators, the influence of nanofillers' anisotropic structures on the actuating performances has been widely reported [7,9,14-16,39-41]. For example, Miyamoto et al. [30] reported a PNIPAMbased hydrogel synthesized by using the shear-force-induced anisotropy of clay nanosheets and subsequent in situ polymerization of PNIPAM, where clay nanosheets were oriented along the applied shear force. Upon heating from 10 to $40^{\circ} \mathrm{C}$, the resultant hydrogel shrank more strongly in the direction perpendicular to the orientation of the clay nanosheets (ca. $60 \%$ shrinkage) than in the parallel direction (ca. 35\% shrinkage) [30]. Hydrogels with an anisotropic structure were also prepared by straightforward orientation of the chains of a polymer network, which were commonly obtained through mechanical compression or stretching, and directional ion

\footnotetext{
${ }^{1}$ School of Science, Beijing Technology and Business University, Beijing 100048, China

${ }^{2}$ Key Laboratory of Bio-Inspired Smart Interfacial Science and Technology of Ministry of Education, School of Chemistry, Beihang University, Beijing 100191, China

${ }^{3}$ Research Institute of Frontier Science, Beihang University, Beijing 100191, China

${ }^{4}$ Beijing Advanced Innovation Center for Biomedical Engineering, Beihang University, Beijing 100191, China

${ }^{5}$ School of Physics, Beihang University, Beijing 100191, China

$\dagger$ These authors contributed equally to this work.

* Corresponding authors (emails: lish@th.btbu.edu.cn (Li S); liumj@buaa.edu.cn (Liu M))
} 
diffusion methods. Inspired by biomaterials, anisotropic hydrogels with oriented polymer networks have potentials to be applied in actuators [7-9]. However, improving the actuating capability by the PNIPAM aligned polymer networks remains a challenge.

Herein, we report an anisotropic composite hydrogel prepared by the mechanical-strain-induced method. A semi-interpenetrating polymer network (semi-IPN) hydrogel of PNIPAM, sodium alginate and carbon nanotubes (PNIPAM/SA/CNTs) was prepared. Then, the sample was physically crosslinked into full-IPN by loading $\mathrm{Ca}^{2+}$ under stretching condition. Since double polymer networks and CNTs in hydrogels were aligned by stretching, the resultant hydrogels showed anisotropic structures and related behaviors. The tensile strength of the anisotropic composite hydrogels along the stretched direction was four times higher than that of the isotropic composite hydrogels. Microscopic anisotropic structures also endowed the composite hydrogel with macroscopic thermo-responsive anisotropic deformation properties. When the temperature reached above the LCST, the anisotropic composite hydrogel showed 50\% contraction strain along the stretched direction, while only $25 \%$ in the perpendicular direction. The hydrogel showed capabilities to lift 100 times its weight with $20 \%$ contraction strain along the stretched direction, which showed comparable actuation behavior to human skeletal muscles ( 20\%) [42-47]. Based on these performances, a bilayer hydrogel was designed to bend and twine with maximum of $390^{\circ}$, which is capable of mimicking the tendril behavior of plants.

\section{RESULTS AND DISCUSSION}

\section{Construction of anisotropic composite hydrogels via mechanical-strain-induced method}

The anisotropic composite hydrogels were prepared through two steps, as shown in Fig. 1, and the operating procedure is shown in Fig. S1a. At first, the semi-IPN composite hydrogels were prepared, where NIPAM monomer and $N, N^{\prime}$-methylenebisacrylamide (BIS) were initiated by potassium persulfate (KPS) to copolymerize at a specific ratio and then crosslinked in the presence of sodium alginate (SA) and CNTs (Fig. 1a). In the second step, the semi-IPN hydrogels were stretched to a given strain and then soaked in a solution of $\mathrm{Ca}^{2+}\left(0.3 \mathrm{~mol} \mathrm{~L}^{-1}\right)$. When the semi-IPN hydrogels were transformed into full-IPN hydrogels, the samples were immersed in deionized water to remove excess $\mathrm{Ca}^{2+}$.

The semi-IPN composite hydrogels are composed of a
PNIPAM polymer network, and BIS plays a crosslinking role in the copolymerization, where a low crosslinking degree of PNIPAM polymer network is necessary to be controlled by the content of BIS (Table S1). The semi-IPN composite hydrogels contain the alginate polymer chains. Alginate, a natural polysaccharide derived from brown sea-weeds, is a polyanionic linear copolymer composed of mannuronic acid ( $M$ unit) and guluronic acid ( $\mathrm{G}$ unit). In an aqueous solution, the $\mathrm{G}$ blocks of guluronic acid in the dissolved alginate polymer chains form the ionic crosslinks through divalent cations (for example, $\mathrm{Ca}^{2+}$, calcium alginate (CA)), resulting in a crosslinked polymer network [48-51]. As the semi-IPN composite hydrogel is composed of a PNIPAM polymer network with low crosslinking degree and alginate polymer chains, it is possible to stretch the sample and maintain it at a large deformation. Under these circumstances, $G$ blocks were drawn closer under stretching and preferentially chelated with $\mathrm{Ca}^{2+}$ to fix the strain of double polymer networks. It demonstrated the retention rate of strain (Fig. S1a, b) decreased rapidly when the given strain reached above $200 \%$, as CA crosslinking polymer networks were inefficient to fix the double polymer networks owing to the elasticity of polymer chains under high strain. Therefore, the given strain of the anisotropic composite hydrogel was set to $200 \%$. The formation of a secondary physical crosslinking network could serve as an energy dissipation source, which improved the overall mechanical performances of the hydrogels $[27,48,52-55]$. The addition of CNTs into the hydrogels not only improved their performances in terms of mechanical properties but also endowed them with the ability of near-infrared (NIR) actuation [52,56-58]. Based on these, the anisotropic composite hydrogels (CNTs-PNIPAM/CA (A)) containing CNTs were prepared, along with the isotropic composite hydrogels (CNTs-PNIPAM/CA (I)) containing CNTs, anisotropic composite hydrogels (PNIPAM/CA (A)) without CNTs, and the isotropic composite hydrogels (PNIPAM/CA (I)) without CNTs which were used as control.

\section{Morphological characterization}

The microscopic morphologies of the isotropic and anisotropic composite hydrogels were characterized by field emission scanning electron microscopy (FESEM) and polarized optical microscopy (POM). As shown in Fig. $1 b(i), c(i)$ and Fig. S2a(i), b(i), the FESEM images obtained from the cross-sections of the hydrogels indicate that the isotropic hydrogels exhibit random and uniform porous structures. In contrast, the anisotropic composite 


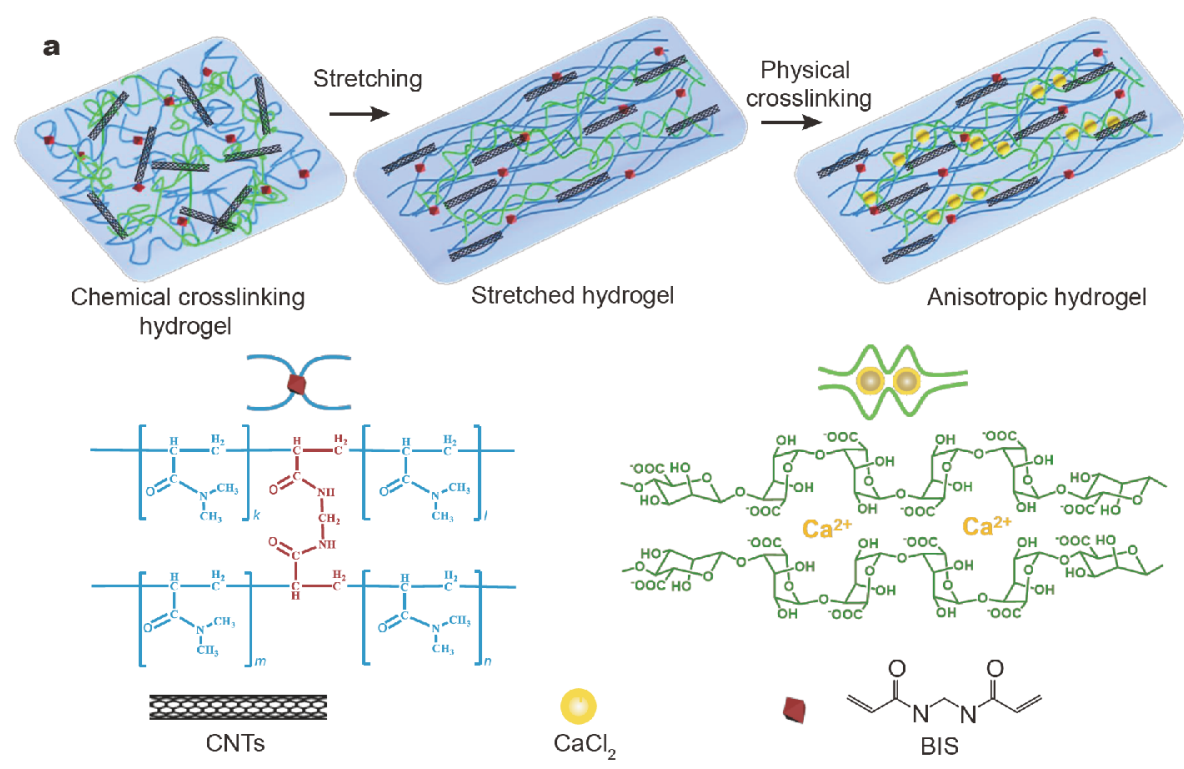

b

PNIPAM/CA (I)
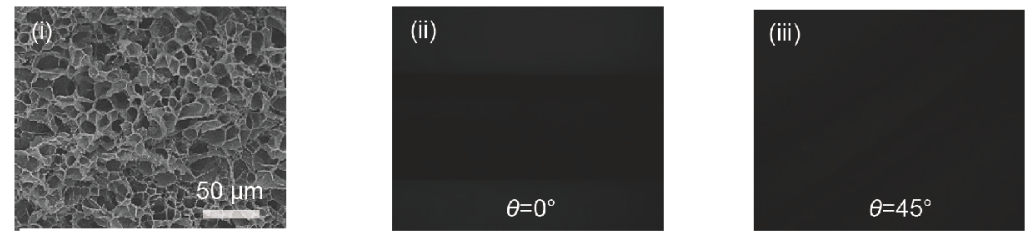

c

PNIPAM/CA (A)
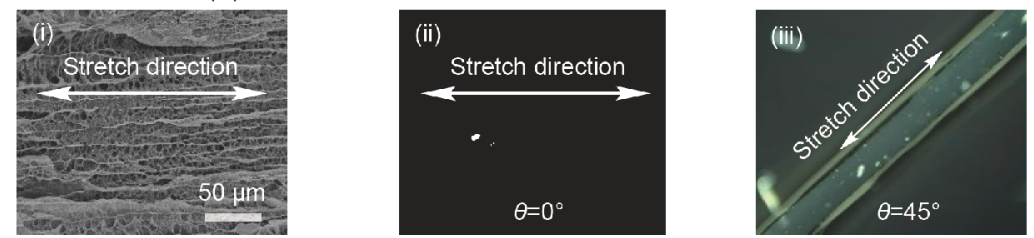

d
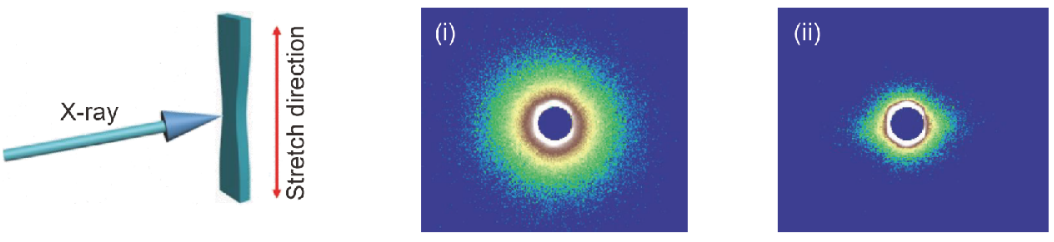

CNTs-PNIPAM/CA (I)

CNTS-PNIPAM/CA (A)

Figure 1 (a) Schematic description of the anisotropic hydrogel. NIPAM, SA, CNTs, covalent crosslinkers (BIS) for NIPAM, and ionic crosslinkers (calcium chloride) for SA. (b) (i) FESEM image of the fractured PNIPAM/CA (I) hydrogel; (ii, iii) polarized microscope images of the PNIPAM/CA (I) hydrogel; (c) (i) FESEM image of the fractured PNIPAM/CA (A) hydrogels; (ii, iii) polarized microscope images of the PNIPAM/CA (A) hydrogel; (d) 2D SAXS images of CNTs-PNIPAM/CA (I) and CNTs-PNIPAM/CA (A) hydrogels.

hydrogels consist of oriented hole-like aisles in size of microns.

This change of structures demonstrated that the inner structures of hydrogels were altered significantly through the treatment by the mechanical-strain-induced method. As confirmed by POM observation, the anisotropic composite hydrogels showed an anisotropic structure along the stretched direction (Fig. 1c(ii, iii), Figs S2b(ii, iii) and S3b)). Under crossed nicol, a complete extinction was observed when the azimuthal angle between the polarized direction of incident light and stretched direction was $0^{\circ}$, while the maximum brightness was observed at $45^{\circ}$ [22,59]. Therefore, changes in dark and bright (birefringence behavior) images were observed by rotating the anisotropic composite hydrogels at an interval of $45^{\circ}$. On the other hand, the POM image of the isotropic 
composite hydrogels showed unirefringence behavior (dark) (Fig. 1b(ii, iii), Figs S2a(ii, iii) and S3a). These results demonstrated that the oriented polymer networks were built by the mechanical-strain-induced method.

To better understand the orientation of CNTs in the hydrogels, small-angle X-ray scattering (SAXS) measurements were carried out [22]. The schematic illustration (Fig. 1d) shows the hydrogel is exposed to an X-ray beam from the orthogonal to the stretching direction. The resultant $2 \mathrm{D}$ patterns of the SAXS measurements on the hydrogels with different strains are given in Fig. 1d and Fig. S2c. In the SAXS patterns of CNTs-PNIPAM/CA (I) and CNTs-PNIPAM/CA (A) hydrogels, an anisotropic scattering parallel to the equator emerged in the CNTsPNIPAM/CA (A) hydrogel. The scattering patterns did not change in the PNIPAM/CA (I) and PNIPAM/CA (A) hydrogels, as shown in Fig. S2c. These results demonstrate that the anisotropic scattering of CNTs-PNIPAM/ CA (A) originates from the orientation of CNTs. Also, they indicate that CNTs orient in parallel to the direction by the mechanical-strain-induced method.

\section{Studies on mechanical properties}

In the mechanical-strain-induced method, a high tensile fracture strain of hydrogel polymer network is required to induce strain further $[18,22]$. Unfortunately, traditional PNIPAM hydrogels with single network cannot meet such demand owing to their weak and fragile nature. In recent studies, semi-IPN PNIPAM-based hydrogels have shown a possible way to overcome this problem. Alginate was chosen to form the second polymer network, and the addition of SA could improve the mechanical properties of the PNIPAM-based hydrogel $[36,60]$. The ratios of components (PNIPAM/SA) were explored to meet the requirement of the mechanical-strain-induced method, and the results displayed that the alginate polymer network had a significant impact on the tensile strain of semi-IPN PNIPAM/SA hydrogels. When the weight ratio of PNIPAM (16.95 wt.\%) and SA (1.695 wt.\%) reached 10:1 (Tables S1 and S2), the sample presented the highest tensile strain of $750 \%$, which was three times higher than that of PNIPAM hydrogels with a single polymer network (Fig. S4a, b).

It is noteworthy to mention that the mechanical properties of PNIPAM/CA full-IPN hydrogels are different from their parents, CA and PNIPAM hydrogels, respectively. As shown in Fig. S4a, the tensile strengths of PNIPAM, CA and PNIPAM/CA are 1, 383 and $120 \mathrm{kPa}$, respectively. This reveals that the overall mechanical performance of PNIPAM/CA could be largely attributed to the addition of CA as the second crosslinked polymer network, which could dissipate energy under large deformation $[48,61,62]$.

To verify the anisotropic mechanical properties of PNIPAM/CA (A) hydrogels, the tensile stresses of the asprepared hydrogels in parallel and perpendicular to the stretched directions were measured. Also, the tensile stress of the isotropic hydrogels was measured, and the obtained data are given in Fig. S4f. It could be noted that the anisotropic composite hydrogels in parallel to the stretched direction displayed much higher tensile stress compared with others. The tensile strength of the isotropic and anisotropic composite hydrogels in perpendicular to the stretched direction were 0.08 and $0.12 \mathrm{MPa}$, respectively, while the tensile strength along the stretched direction of the anisotropic composite hydrogel reached $0.27 \mathrm{MPa}$. The tensile stress-strain curve of the isotropic composite hydrogels increased rapidly and then suffered from an apparent yield phenomenon. However, the tensile stress-strain curve of the anisotropic composite hydrogels increased with the tensile strain rapidly, and a slighter yield was found. These results indicated that the CA in the composite hydrogels improved their mechanical properties, and the aligned CA polymer networks led to anisotropic mechanical performances.

The addition of CNTs showed enormous improvement to the tensile stress of the anisotropic composite hydrogels in parallel to the stretched direction, and the tensile stress increased linearly with an increase in the amount of CNTs (Fig. 2a-c). The tensile strength of the CNTsPNIPAM/CA (A) hydrogels parallel to the stretched direction reaches $0.9 \mathrm{MPa}$ at the highest CNTs concentration, which is about three times higher than PNIPAM/CA (A) hydrogels (Fig. 2c and Fig. S4e). Interestingly, the addition of CNTs showed an insignificant effect on the tensile stress of the isotropic composite hydrogels, as well as that of the anisotropic composite hydrogels in perpendicular to the stretched direction. As shown in Fig. 2b, the tensile strength of the CNTs-PNIPAM/CA (A) hydrogels is four times higher than that of CNTs-PNIPAM/ CA (I) hydrogels. This could be due to that the ultrapure CNTs used in this work were dispersed with the addition of nonionic surfactants, whose hydrophobic ends were benzene rings, and hydrophilic ends were hydroxyl groups. The hydrophobic benzene rings of the nonionic surfactants can form a $\pi-\pi$ conjugate with the CNTs [52]. The hydroxyl groups not only assist CNTs to disperse in the aqueous solution, but also facilitate the formation of hydrogen bonds with hydroxyl or carboxyl groups of the polymer network in hydrogels [56,63]. Due to the hy- 
a
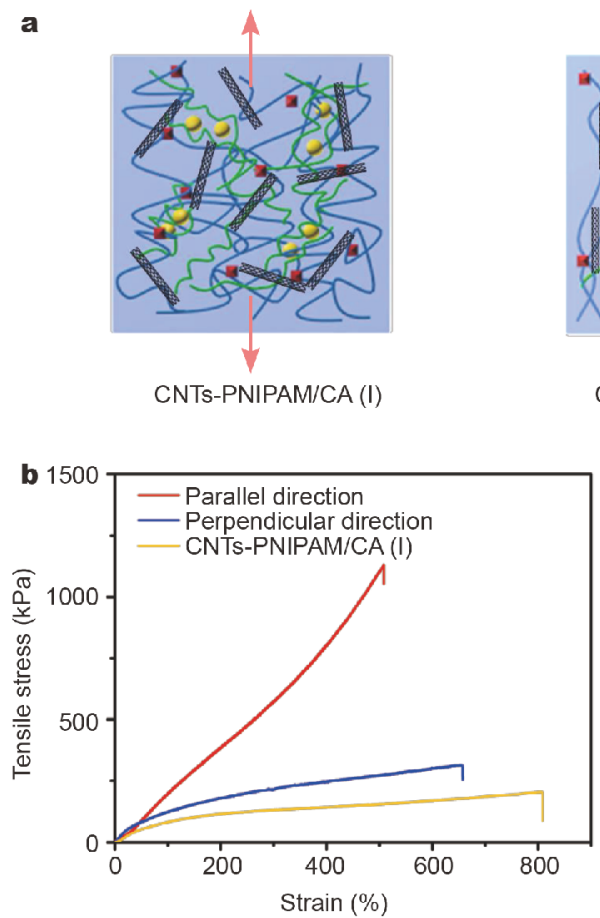
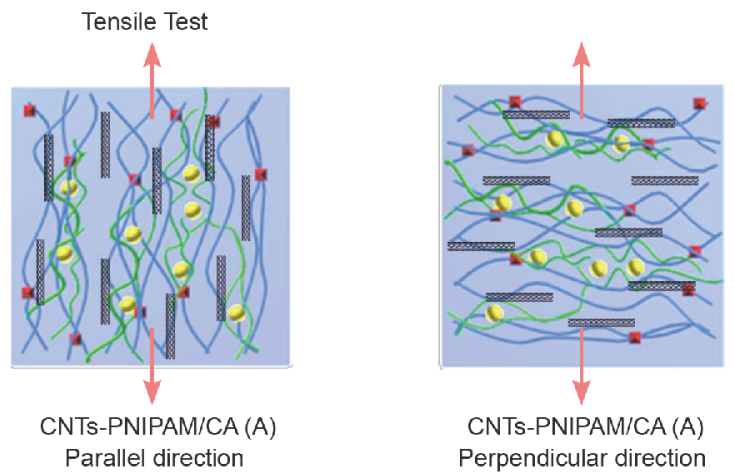

CNTS-PNIPAM/CA (A) Perpendicular direction

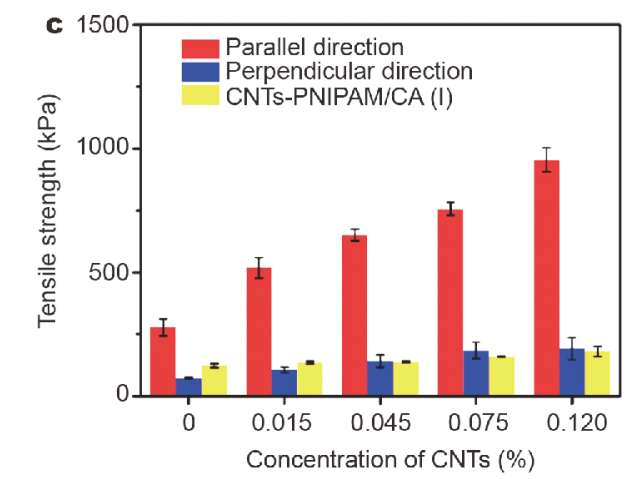

Figure 2 The mechanical properties of the hydrogels. (a) Schematic representation for the mechanical tests of the samples; (b) tensile strain-stress curves of CNTs-PNIPAM/CA (I) hydrogel (yellow line) and CNTs-PNIPAM/CA (A) hydrogel (red line for the parallel direction, blue line for the perpendicular direction); (c) the tensile strengths of hydrogels with different concentrations of CNTs.

drogen bonding of polymer networks with CNTs, the ordered arrangement of CNTs further enhanced the mechanical properties significantly in the stretched direction [32].

\section{Contraction analysis upon LCST}

It is well known that the phase transition of the PNIPAMbased hydrogels will induce contraction of volume change, but the contraction speed is rather slow $[37,64,65]$. In our experiments, the contraction of the samples also occurred above the LCST and showed anisotropic behavior with a rapid thermal response. To investigate the thermal contraction of the anisotropic and isotropic composite hydrogels, bulk-like hydrogel samples with width $\times$ length $\times$ thickness of $10 \mathrm{~mm} \times 10 \mathrm{~mm} \times$ $1 \mathrm{~mm}$ were prepared, as shown in Fig. $3 \mathrm{a}(\mathrm{i}), \mathrm{b}(\mathrm{i})$ and Fig. S5a, b. When the samples were heated to $45^{\circ} \mathrm{C}$, the isotropic composite hydrogels showed isotropic volume changes (Fig. 3a(i) and Fig. S5a), while the anisotropic composite hydrogels contracted unevenly from square to a rectangle (Fig. 3b(i) and Fig. S5b). The thermal response rate tests are shown in Fig. 3a(ii, iii) and b(ii, iii), and it could be seen that the CNTs-PNIPAM/CA (A) hydrogels contracted 50\% along the stretched direction and $25 \%$ in the perpendicular direction under $45^{\circ} \mathrm{C}$ in $60 \mathrm{~s}$, while the CNTs-PNIPAM/CA (I) hydrogels only showed $20 \%$ isotropic contraction strain. These results indicated that the PNIPAM polymer networks provided thermo-responsive deformation, and the orientation of the polymer networks caused anisotropic deformation.

The results of weight loss experiments, as shown in Fig. 3c, indicated that the CNTs-PNIPAM/CA (A) hydrogels lost about $75 \%$ of the total weight at $45^{\circ} \mathrm{C}$ in $60 \mathrm{~s}$, while the CNTs-PNIPAM/CA (I) hydrogels only lost $50 \%$. The remaining weight of both samples gradually changed to the solid content of these hydrogels. These results suggested that by applying PNIPAM-based hydrogels with anisotropic structures, the thermal response properties enhanced significantly. This phenomenon could be explained by studying the inner structure of hydrogels as well as the orientation of the double polymer chains. The inner structures of anisotropic composite hydrogels changed from random and uniform porous structures into oriented hole-like aisles in micron (Fig. 1b, $c$ and Fig. S5a, b). This structure facilitated the discharge of water along the direction of oriented aisles during 

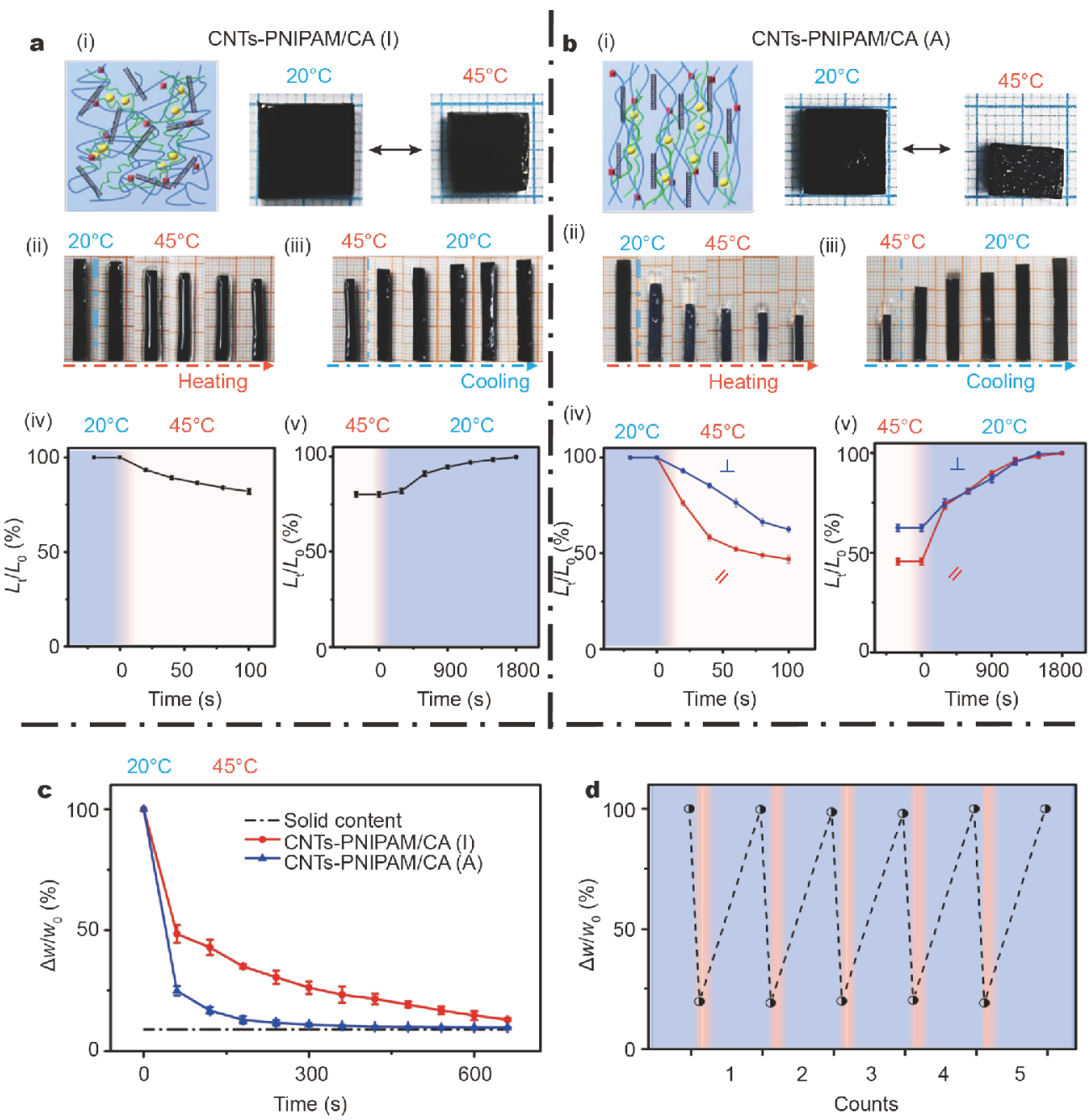

Figure 3 Thermo-responsive behavior of the hydrogels. (a) (i) Schematic representation and pictures of CNTs-PNIPAM/CA (I) hydrogel (CNTs, 0.12 wt.\%); (ii-v) pictures of CNTs-PNIPAM/CA (I) hydrogel and plots showing the changes in the relative length $\left(L_{t} / L_{0}(\%)\right.$ ). (b) (i) Schematic representation and pictures of CNTs-PNIPAM/CA (A) hydrogel; (ii-v) pictures of CNTs-PNIPAM/CA (A) hydrogel and plots showing the changes in the relative length $\left(L_{t} / L_{0}(\%)\right)$. (c) Plots showing changes in the relative weight of hydrogels $\left(\Delta w / w_{0}(\%)\right) v s$. time. (d) Reversibility of CNTsPNIPAM/CA (A) hydrogel. $L_{0}$ is the original length of hydrogels, $L_{\mathrm{t}}$ is the length of the contracted hydrogels. $\Delta w=w_{0}-w_{\mathrm{t}}, w_{0}$ is the original weight of hydrogels, and $w_{\mathrm{t}}$ is the weight of the contracted hydrogels.

volume change above the LCST and accelerated weight loss.

Furthermore, the reversible deformation of the CNTsPNIPAM/CA (A) hydrogel was characterized. When cooled below the LCST, the hydrogel absorbed water again and recovered to its original size. Since swelling process is a relatively slow step [64], the CNTs-PNIPAM/ CA (A) hydrogel with $75 \%$ of the weight loss required more than $30 \mathrm{~min}$ to recover when cooling was carried out in a water bath $\left(20^{\circ} \mathrm{C}\right)$. This process was repeated five times, and no significant changes were ascertained (Fig. 3d), representing good reversibility of thermo-responsive anisotropic deformation.

\section{Actuators by thermal-stimulus}

By taking advantage of samples with rapid thermal shape change, high contraction strain, as well as high mechanical performance, a hydrogel actuator that was driven by warm water or NIR was designed (Fig. S5c, d, Fig. 4a, b and Movies S1-S7). In the case of actuating with warm water, the loading of the samples with different weights was considered in warm water $\left(45^{\circ} \mathrm{C}\right)$ to test the actuating ability. As shown in Fig. S5c, the CNTs-PNIPAM/CA (A) hydrogel lifted a load ten times its weight, and the actuation of the samples along the stretched direction could reach above $45 \%$ of the contraction strain in $60 \mathrm{~s}$ (Movie S1). Moreover, because of the enhanced mechanical 
a

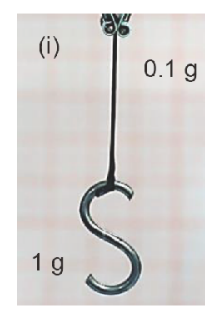

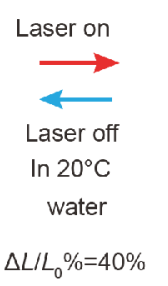

\section{$0^{\circ}$}

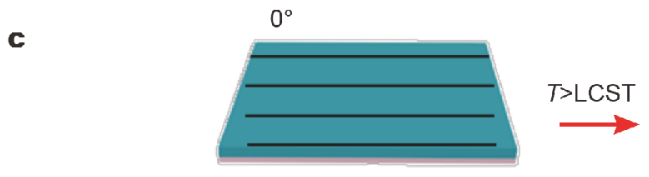

b

Lift up a load 100 times its own weight

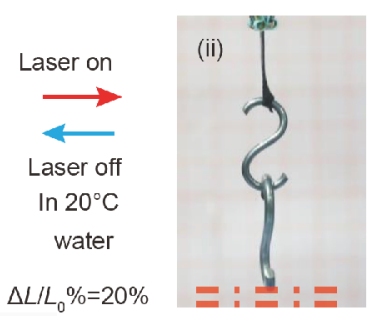

$3 \mathrm{~g}$
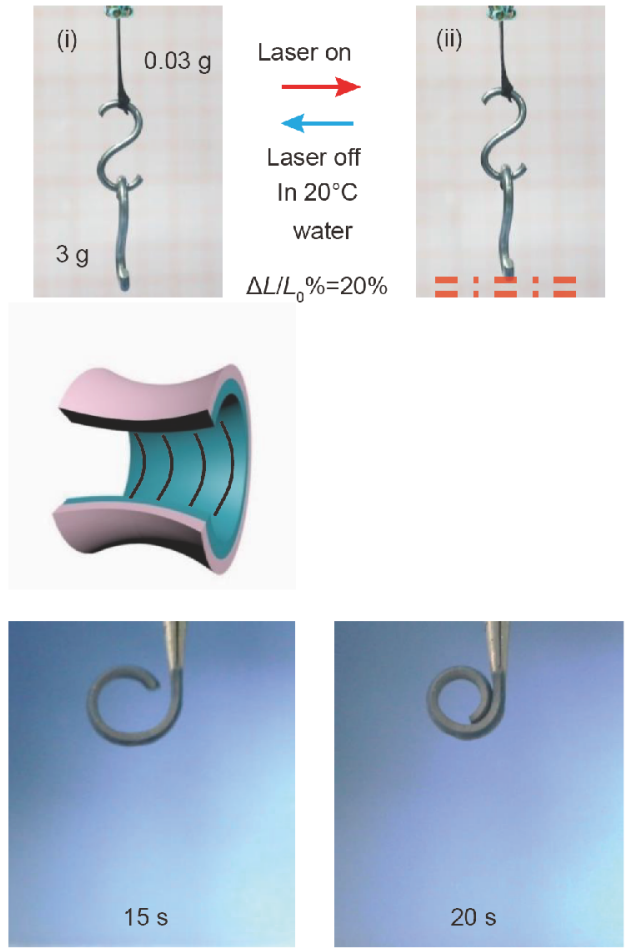

$8 \mathrm{~s}$
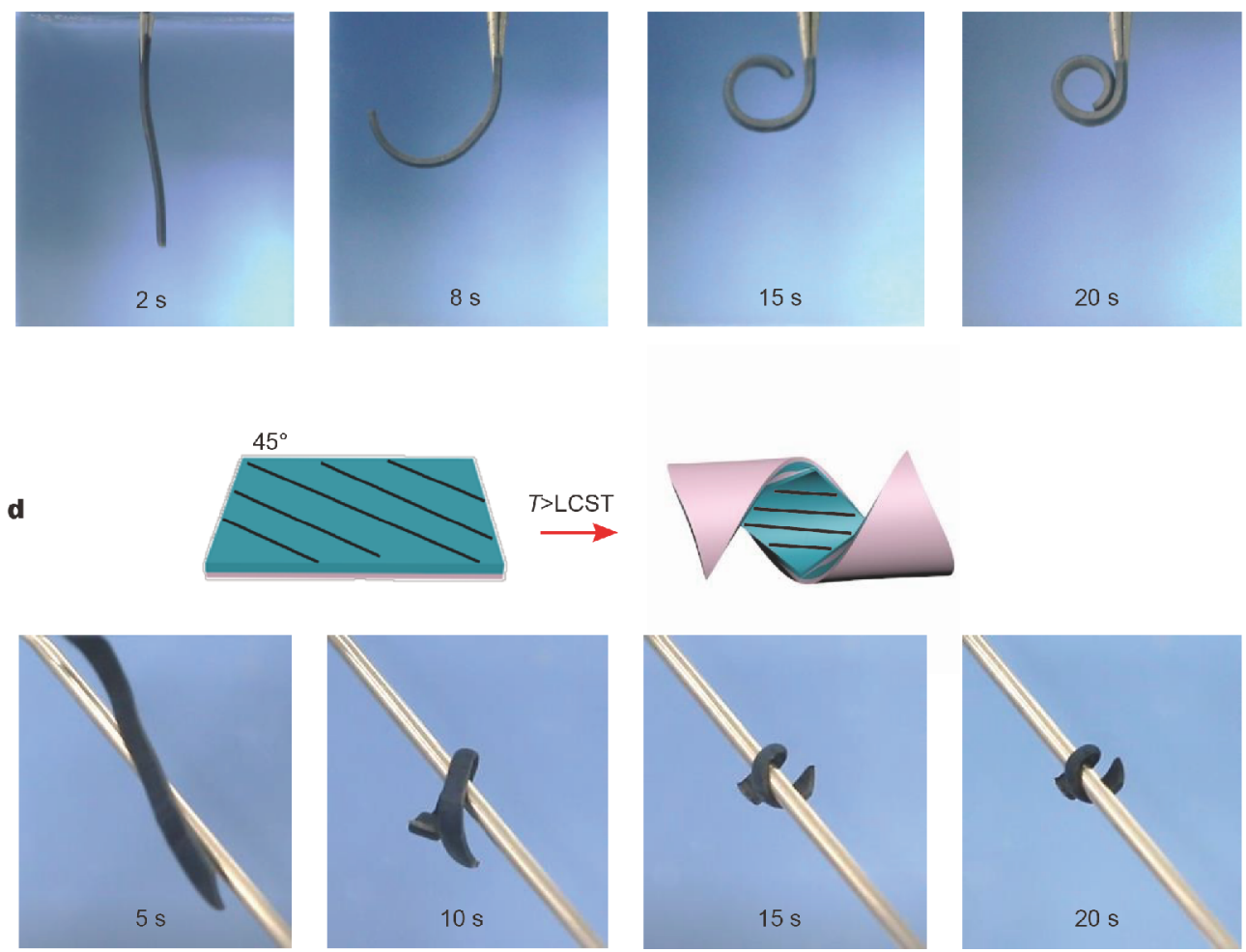

Figure 4 Actuation of hydrogels. Pictures of the lifting weight by NIR: (a) $m_{\mathrm{w}}=0.1 \mathrm{~g}, m_{\mathrm{H}}=1 \mathrm{~g}, \Delta L / L_{0}=40 \%$; $(\mathrm{b}) m_{\mathrm{w}}=0.03 \mathrm{~g}, m_{\mathrm{H}}=3 \mathrm{~g}, \Delta L / L_{0}=20 \%$. Contraction strain $=\Delta L / L_{0}(\%), \Delta L=L_{0}-L_{\mathrm{t}}, L_{0}$ is the original length of the hydrogel, and $L_{\mathrm{t}}$ is the length of the contracted hydrogel. Actuations of Bi- $0^{\circ}$ (c) and $\mathrm{Bi}-45^{\circ}(\mathrm{d})$ in water at $80^{\circ} \mathrm{C}$.

properties of CNTs-PNIPAM/CA (A) hydrogels, the length of the sample had no distinct elongation when a load of 100 times its weight was added. When the sample was immersed in warm water $\left(45^{\circ} \mathrm{C}\right)$ and the loading was 100 times its weight, it showed comparable contraction to human skeletal muscles with $20 \%$ strain along the actuated direction (Movie S2 and Fig. S5d) [42-47].

Since CNTs have a high absorption of NIR light and high efficiency of thermal transformation $[53,57,58]$, the anisotropic composite hydrogels also showed excellent actuation performances. As shown in Fig. S6a and b, with an increase in the concentration of CNTs, the temperature increased by NIR and thus the sample could be quickly heated above the LCST. Therefore, the heat that CNTs converted by NIR could induce the phase transition of anisotropic composite hydrogels instantaneously. 
As shown in Movies S3, S4 and Fig. 4a, b, NIR lightdriven tests showed the same deformation performance as in warm water, but the former had a shorter driving time than the latter. This could be due to that CNTs acted as a "molecular heater" to raise the local temperature of the composite hydrogel via high photo-thermal conversion [58], resulting in high heating rates than warm water.

The thermo-responsive anisotropic deformation properties of CNTs-PNIPAM/CA (A) hydrogels were used to design an actuator, which had a bilayer of two structural hydrogel components with different contraction abilities. One layer was CNTs-PNIPAM/CA (A) hydrogel, which contracted $50 \%$ along the stretched direction; another layer was PNIPAM hydrogel, which contracted $30 \%$ in both directions (Table S3). As shown in Fig. S7a, the resulting samples were cut at different angles to provide samples parallelled to the stretched direction $\left(\mathrm{Bi}-0^{\circ}\right)$, and $45^{\circ}$ to the stretched direction $\left(\mathrm{Bi}-45^{\circ}\right)$. When the sample $\mathrm{Bi}-0^{\circ}$ was immersed in water at $80^{\circ} \mathrm{C}$, the sample showed rolling along the stretched direction with the random PNIPAM layer forming outside of the rolls due to its differential contraction strain (Fig. S7b). Fig. 4c shows the images of the $\mathrm{Bi}-0^{\circ}$ hydrogel in water at $80^{\circ} \mathrm{C}$, and the rolling angle of $\mathrm{Bi}-0^{\circ}$ sample changed from $0^{\circ}$ to $390^{\circ}$ rapidly in the first $20 \mathrm{~s}$ under heating (Fig. S7b and Movie S5). These changes in rolling angle showed an enhanced actuation capability of this material. It is known that tendril climber plants can extend and twine around the surrounding support using their helical tendrils. A similar phenomenon was found by heating the $\mathrm{Bi}-45^{\circ}$ composite hydrogel in water at $80^{\circ} \mathrm{C}$, where precisely the planar sheet transformed into a helix that mimicked plant tendrils, as shown in Fig. 4d and Movie S6. It is due to that the asymmetric internal stress in the composite sheet was built by different contraction strain of layers [66].

\section{CONCLUSIONS}

In summary, with the combination of IPN and mechanical-strain-induced method, anisotropic composite hydrogels with a high actuating performance enhanced by the alignment of double polymer networks were successfully prepared. The CNTs-PNIPAM/CA (A) hydrogel achieved a mechanical strength of $0.9 \mathrm{MPa}$ along the stretched direction, and the thermal-response contraction strain of the anisotropic hydrogel reached 50\% along the stretched direction. This study demonstrated the enhanced actuating abilities of anisotropic composite hydrogels, which could lift 100 times of their own weight in less than $20 \mathrm{~s}$. Moreover, a bilayer hydrogel (PNIPAM/ CNTs-PNIPAM/CA (A)) can bend and twine around the rachis of the surrounding support by tailoring at a specific angle. These advantages endowed the anisotropic hydrogel composite with a wide range of applications, including artificial muscles and soft tissues. This study provides new insight into improving the actuating abilities of hydrogels with aligned polymer networks.

\section{Received 15 November 2019; accepted 18 December 2019;}

published online 18 January 2020

1 Liu M, Jiang L. Dialectics of nature in materials science: Binary cooperative complementary materials. Sci China Mater, 2016, 59: 239-246

2 Che L, Liu YX, Zhao ZG, et al. Design and preparation of bionic multi-scale functional hydrogels: From 2D interfaces to 3D networks. Acta Polym Sin, 2018, 9: 1155-1174

3 Matsumoto A, Yoshida R, Kataoka K. Glucose-responsive polymer gel bearing phenylborate derivative as a glucose-sensing moiety operating at the physiological pH. Biomacromolecules, 2004, 5: 1038-1045

4 Nagase K, Kobayashi J, Okano T. Temperature-responsive intelligent interfaces for biomolecular separation and cell sheet engineering. J R Soc Interface, 2009, 6: S293-309

5 Okano T, Yamada N, Sakai H, et al. A novel recovery system for cultured cells using plasma-treated polystyrene dishes grafted with poly( $N$-isopropylacrylamide). J Biomed Mater Res, 1993, 27: 12431251

6 Yamato M, Okano T. Cell sheet engineering. Mater Today, 2004, 7: 42-47

7 Zhao Z, Fang R, Rong Q, et al. Bioinspired nanocomposite hydrogels with highly ordered structures. Adv Mater, 2017, 29: 1703045-1703061

8 Le $\mathrm{X}, \mathrm{Lu} \mathrm{W}$, Zhang J, et al. Recent progress in biomimetic anisotropic hydrogel actuators. Adv Sci, 2019, 6: 1801584-1801598

9 Sano K, Ishida Y, Aida T. Synthesis of anisotropic hydrogels and their applications. Angew Chem Int Ed, 2018, 57: 2532-2543

10 Morales D, Bharti B, Dickey MD, et al. Bending of responsive hydrogel sheets guided by field-assembled microparticle endoskeleton structures. Small, 2016, 12: 2283-2290

11 Ramón-Azcón J, Ahadian S, Estili M, et al. Dielectrophoretically aligned carbon nanotubes to control electrical and mechanical properties of hydrogels to fabricate contractile muscle myofibers. Adv Mater, 2013, 25: 4028-4034

12 Erb RM, Sander JS, Grisch R, et al. Self-shaping composites with programmable bioinspired microstructures. Nat Commun, 2013, 4: 1712

$13 \mathrm{Hu}$ K, Sun J, Guo Z, et al. A novel magnetic hydrogel with aligned magnetic colloidal assemblies showing controllable enhancement of magnetothermal effect in the presence of alternating magnetic field. Adv Mater, 2015, 27: 2507-2514

14 Kim YS, Liu M, Ishida Y, et al. Thermoresponsive actuation enabled by permittivity switching in an electrostatically anisotropic hydrogel. Nat Mater, 2015, 14: 1002-1007

15 Liu M, Ishida Y, Ebina Y, et al. An anisotropic hydrogel with electrostatic repulsion between cofacially aligned nanosheets. Nature, 2015, 517: 68-72

16 Maggini L, Liu M, Ishida Y, et al. Anisotropically luminescent hydrogels containing magnetically-aligned MWCNTs-Eu(III) hybrids. Adv Mater, 2013, 25: 2462-2467

17 Rešetič A, Milavec J, Zupančič B, et al. Polymer-dispersed liquid 
crystal elastomers. Nat Commun, 2016, 7: 13140

18 Lin $\mathrm{P}$, Zhang T, Wang $\mathrm{X}$, et al. Freezing molecular orientation under stretch for high mechanical strength but anisotropic hydrogels. Small, 2016, 12: 4386-4392

19 Matsuda T, Kawakami R, Namba R, et al. Mechanoresponsive selfgrowing hydrogels inspired by muscle training. Science, 2019, 363: 504-508

20 Shibayama M, Karino T, Miyazaki S, et al. Small-angle neutron scattering study on uniaxially stretched poly $(N$-isopropylacrylamide)-clay nanocomposite gels. Macromolecules, 2005, 38: 10772-10781

21 Murata K, Haraguchi K. Optical anisotropy in polymer-clay nanocomposite hydrogel and its change on uniaxial deformation. J Mater Chem, 2007, 17: 3385

22 Shikinaka K, Koizumi Y, Kaneda K, et al. Strain-induced reversible isotropic-anisotropic structural transition of imogolite hydrogels. Polymer, 2013, 54: 2489-2492

23 Ye D, Yang P, Lei X, et al. Robust anisotropic cellulose hydrogels fabricated via strong self-aggregation forces for cardiomyocytes unidirectional growth. Chem Mater, 2018, 30: 5175-5183

24 Mredha MTI, Guo YZ, Nonoyama T, et al. A facile method to fabricate anisotropic hydrogels with perfectly aligned hierarchical fibrous structures. Adv Mater, 2018, 30: 1704937-1704945

25 Wu ZL, Sawada D, Kurokawa T, et al. Strain-induced molecular reorientation and birefringence reversion of a robust, anisotropic double-network hydrogel. Macromolecules, 2011, 44: 3542-3547

$26 \mathrm{Kim} \mathrm{SH}, \mathrm{Im} \mathrm{SK}$, Oh SJ, et al. Anisotropically organized threedimensional culture platform for reconstruction of a hippocampal neural network. Nat Commun, 2017, 8: 14346

27 Choi S, Kim J. Designed fabrication of super-stiff, anisotropic hybrid hydrogels via linear remodeling of polymer networks and subsequent crosslinking. J Mater Chem B, 2015, 3: 1479-1483

28 Chen Y, Jiao C, Peng X, et al. Biomimetic anisotropic poly(vinyl alcohol) hydrogels with significantly enhanced mechanical properties by freezing-thawing under drawing. J Mater Chem B, 2019, 7: 3243-3249

29 Sydney Gladman A, Matsumoto EA, Nuzzo RG, et al. Biomimetic 4D printing. Nat Mater, 2016, 15: 413-418

30 Miyamoto N, Shintate M, Ikeda S, et al. Liquid crystalline inorganic nanosheets for facile synthesis of polymer hydrogels with anisotropies in structure, optical property, swelling/deswelling, and ion transport/fixation. Chem Commun, 2013, 49: 1082-1084

31 Bai H, Polini A, Delattre B, et al. Thermoresponsive composite hydrogels with aligned macroporous structure by ice-templated assembly. Chem Mater, 2013, 25: 4551-4556

32 Downes R, Wang S, Haldane D, et al. Strain-induced alignment mechanisms of carbon nanotube networks. Adv Eng Mater, 2015, 17: 349-358

33 Inadomi T, Ikeda S, Okumura $\mathrm{Y}$, et al. Photo-induced anomalous deformation of poly( $N$-isopropylacrylamide) gel hybridized with an inorganic nanosheet liquid crystal aligned by electric field. Macromol Rapid Commun, 2014, 35: 1741-1746

34 Yoshida R, Uchida K, Kaneko Y, et al. Comb-type grafted hydrogels with rapid deswelling response to temperature changes. Nature, 1995, 374: 240-242

35 Yan Q, Hoffman AS. Synthesis of macroporous hydrogels with rapid swelling and deswelling properties for delivery of macromolecules. Polymer, 1995, 36: 887-889

36 Dragan ES. Design and applications of interpenetrating polymer network hydrogels. A review. Chem Eng J, 2014, 243: 572-590
37 Xia LW, Ju XJ, Liu JJ, et al. Responsive hydrogels with poly $(N$ isopropylacrylamide-co-acrylic acid) colloidal spheres as building blocks. J Colloid Interface Sci, 2010, 349: 106-113

$38 \mathrm{Xu} \mathrm{XD}$, Zhang XZ, Yang J, et al. Strategy to introduce a pendent micellar structure into poly( $N$-isopropylacrylamide) hydrogels. Langmuir, 2007, 23: 4231-4236

39 Sun Z, Yamauchi Y, Araoka F, et al. An anisotropic hydrogel actuator enabling earthworm-like directed peristaltic crawling. Angew Chem Int Ed, 2018, 57: 15772-15776

40 Zheng SY, Shen Y, Zhu F, et al. Programmed deformations of 3Dprinted tough physical hydrogels with high response speed and large output force. Adv Funct Mater, 2018, 28: 1803366

41 Ma P, Niu B, Lin J, et al. Sequentially controlled deformations of patterned hydrogels into $3 \mathrm{D}$ configurations with multilevel structures. Macromol Rapid Commun, 2019, 40: 1800681

42 Madden JDW, Vandesteeg NA, Anquetil PA, et al. Artificial muscle technology: Physical principles and naval prospects. IEEE J Ocean Eng, 2004, 29: 706-728

43 Haines CS, Lima MD, Li N, et al. Artificial muscles from fishing line and sewing thread. Science, 2014, 343: 868-872

44 Lima MD, Li N, Jung de Andrade M, et al. Electrically, chemically, and photonically powered torsional and tensile actuation of hybrid carbon nanotube yarn muscles. Science, 2012, 338: 928-932

45 Lee JA, Li N, Haines CS, et al. Electrochemically powered, energyconserving carbon nanotube artificial muscles. Adv Mater, 2017, 29: $1700870-1700877$

46 Lee SH, Kim TH, Lima MD, et al. Biothermal sensing of a torsional artificial muscle. Nanoscale, 2016, 8: 3248-3253

47 Aliev AE, Oh J, Kozlov ME, et al. Giant-stroke, superelastic carbon nanotube aerogel muscles. Science, 2009, 323: 1575-1578

48 Sun JY, Zhao X, Illeperuma WRK, et al. Highly stretchable and tough hydrogels. Nature, 2012, 489: 133-136

49 Yang $\mathrm{C}$, Wang $\mathrm{M}$, Haider $\mathrm{H}$, et al. Correction to strengthening alginate/polyacrylamide hydrogels using various multivalent cations. ACS Appl Mater Interfaces, 2013, 5: 13484

50 Yildirim ED, Yin X, Nair K, et al. Fabrication, characterization, and biocompatibility of single-walled carbon nanotube-reinforced alginate composite scaffolds manufactured using freeform fabrication technique. J Biomed Mater Res, 2008, 87B: 406-414

51 Zhao C, Zhang P, Shi R, et al. Super-tough and strong nanocomposite fibers by flow-induced alignment of carbon nanotubes on grooved hydrogel surfaces. Sci China Mater, 2019, 62: 13321340

52 Tan Z, Ohara S, Naito M, et al. Supramolecular hydrogel of bile salts triggered by single-walled carbon nanotubes. Adv Mater, 2011, 23: 4053-4057

53 Islam A, van den Akker A, Feng PXL. Anisotropic thermal conductivity of suspended black phosphorus probed by opto-thermomechanical resonance spectromicroscopy. Nano Lett, 2018, 18: 7683-7691

54 Gong JP, Katsuyama Y, Kurokawa T, et al. Double-network hydrogels with extremely high mechanical strength. Adv Mater, 2003, 15: 1155-1158

55 Yang $\mathrm{CH}$, Wang MX, Haider $\mathrm{H}$, et al. Strengthening alginate/ polyacrylamide hydrogels using various multivalent cations. ACS Appl Mater Interfaces, 2013, 5: 10418-10422

$56 \mathrm{Du} \mathrm{R}, \mathrm{Wu} \mathrm{J}$, Chen L, et al. Hierarchical hydrogen bonds directed multi-functional carbon nanotube-based supramolecular hydrogels. Small, 2014, 10: 1387-1393

57 Pal A, Chhikara BS, Govindaraj A, et al. Synthesis and properties 
of novel nanocomposites made of single-walled carbon nanotubes and low molecular mass organogels and their thermo-responsive behavior triggered by near IR radiation. J Mater Chem, 2008, 18: 2593-2600

58 Fujigaya T, Morimoto T, Niidome $\mathrm{Y}$, et al. NIR laser-driven reversible volume phase transition of single-walled carbon nanotube/ poly $(N$-isopropylacrylamide) composite gels. Adv Mater, 2008, 20: 3610-3614

59 Yang W, Furukawa H, Gong JP. Highly extensible double-network gels with self-assembling anisotropic structure. Adv Mater, 2008, 20: 4499-4503

60 Haq MA, Su Y, Wang D. Mechanical properties of PNIPAM based hydrogels: A review. Mater Sci Eng-C, 2017, 70: 842-855

61 Zhang H, Zhang Y, He L, et al. Thermal-responsive poly $(N$-isopropyl acrylamide)/sodium alginate hydrogels: Preparation, swelling behaviors, and mechanical properties. Colloid Polym Sci, 2016, 294: 1959-1967

62 Gong JP. Why are double network hydrogels so tough? Soft Matter, 2010, 6: 2583

63 Shin MK, Lee B, Kim SH, et al. Synergistic toughening of composite fibres by self-alignment of reduced graphene oxide and carbon nanotubes. Nat Commun, 2012, 3: 650

64 Sato Matsuo E, Tanaka T. Kinetics of discontinuous volume-phase transition of gels. J Chem Phys, 1988, 89: 1695-1703

65 Maeda Y, Higuchi T, Ikeda I. Change in hydration state during the coil-globule transition of aqueous solutions of poly $(\mathrm{N}$-isopropylacrylamide) as evidenced by FTIR spectroscopy. Langmuir, 2000, 16: 7503-7509

66 Thérien-Aubin H, Wu ZL, Nie Z, et al. Multiple shape transformations of composite hydrogel sheets. J Am Chem Soc, 2013, 135: 4834-4839

Acknowledgements This work was financially supported by the National Key R\&D Program of China (2017YFA0207800), the National Natural Science Foundation of China (KZ75006801), the National Natural Science Funds for Distinguished Young Scholars (21725401), the 111 Project (B14009), and Beijing Technology and Business University 2019 postgraduate research capacity improvement plan.

Author contributions Tang P, Chen L, Li S and Liu M designed the project. Tang P and Yan H analyzed the results. Tang P, Yan H, Li S and Liu $\mathrm{M}$ wrote the paper. All authors contributed to the general discussion of the article.

Conflict of interest The authors declare that they have no conflict of interest.

Supplementary information Experimental details and supporting data are available in the online version of the paper.

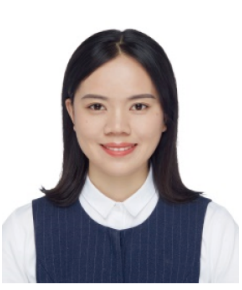

Ping Tang is currently a master student at Beijing Technology and Business University (BTBU). She received her BSc degree in Beijing Institute of Fashion Technology in 2015. Her current research interests focus on the applications of bioinspired gel materials.

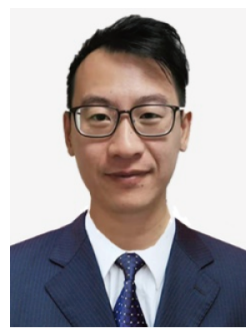

Hao Yan is now a postdoctoral fellow in Prof. Mingjie Liu's group at Beihang University. In 2006, he joined Professor Zhong Zhang's research team and received his $\mathrm{PhD}$ from the National Center for Nanoscience and Technology, Chinese Academy of Sciences in 2011. After that, he worked at Sinopec Beijing Research Institute of Chemical Industry from 2011 to 2018. His current research interests focus on bioinspired surface and hydrogel actuator designs.

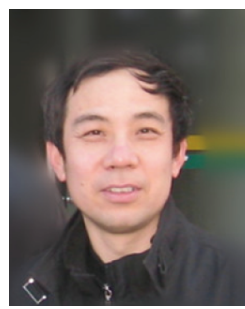

Shuhong Li joined BTBU in 2005 and now he is a professor at BTBU. He received his $\mathrm{PhD}$ degree from the Institute of Chemistry, Chinese Academic Sciences in 2003 under the supervision of Prof. Lei Jiang. He carried out his postdoctoral research in an ERATO project of Japan Science and Technology corporation and in Prof. Benzhong Tang's group at the Hong Kong University of Science and Technology, respectively. His research is mainly on the development and exploration of functional molecules/materials and their advanced properties.

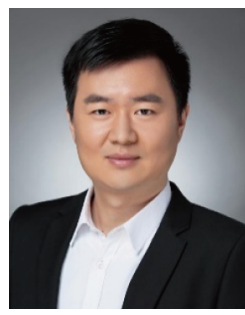

Mingjie Liu is currently a full-time professor at Beihang University. In 2005, he joined Prof. Lei Jiang's group and received his $\mathrm{PhD}$ degree from the National Center for Nanoscience and Technology, Chinese Academy of Sciences (2010). He then worked as a postdoctor in Prof. Takuzo Aida's group in Riken in Japan from 2010 to 2015. In 2015, he was awarded the "1000 Youth Plan program" and joined Beihang University. In 2017, he was awarded the National Natural Science Funds for Distinguished Young Scholars. His current research interests focus on anisotropic soft matter with ordered structures, bioinspired design, and application of gel materials.

\section{一种通过取向聚合物网络提高驱动性能的各向异 性纳米复合水凝胶}

唐萍 $^{1 \dagger}$, 严昊 ${ }^{2,4 \dagger}$, 陈列 ${ }^{2,5}$, 吴青山 ${ }^{2}$, 赵天艺 ${ }^{2}$, 李书宏 ${ }^{*}$, 高海南 ${ }^{1}$, 刘明㷊 ${ }^{2,3^{*}}$

摘要 各向异性复合水凝胶在驱动器和传感器材料领域具有广泛 的应用. 本研究报道了一种通过机械应变诱导方法制备的各向异 性复合水凝胶. 包含聚( $N$-异丙基丙烯酰胺)(PNIPAM) 和海藻酸钠 (SA)的聚合物网络和碳纳米管 $(\mathrm{CNTs})$ 被拉伸同时取向, 然后由非 共价键物理交联固定这种取向结构. 具有双重取向聚合物网络的 复合水凝胶显示出各向异性的光学和机械性能. 与各向同性复合 水凝胶相比, 各向异性复合水凝胶的驱动性能得到了提高, 它能够 以 $20 \%$ 的收缩形变提起自身重量 100 倍的重物. 此外, 制备的双层水 凝胶最大可弯曲 $390^{\circ}$, 并且能够模仿植物的卷须缠绕支撑物. 